\title{
e-Phaïstos
}

e-Phaïstos

Revue d'histoire des techniques / Journal of the history

of technology

VI-1 2017 | 2018

Varia

\section{Le Chantier 1810 du Musee National des Arts et Traditions Populaires (1942-1946) : un conservatoire des techniques et de leurs gestes?}

The 1810 Project of the MNATP (1942-1946): a Conservatory for the Technical Gesture?

\section{Bénédicte Rolland -Villemot}

\section{OpenEdition}

\section{Journals}

Édition électronique

URL : http://journals.openedition.org/ephaistos/2468

DOI : 10.4000/ephaistos.2468

ISSN : 2552-0741

\section{Éditeur}

IHMC - Institut d'histoire moderne et contemporaine (UMR 8066)

Référence électronique

Bénédicte Rolland -Villemot, « Le Chantier 1810 du Musee National des Arts et Traditions Populaires (1942-1946) : un conservatoire des techniques et de leurs gestes? », e-Phaïstos [En ligne], VI-1 2017 | 2018, mis en ligne le 25 juin 2018, consulté le 20 avril 2019. URL : http:// journals.openedition.org/ephaistos/2468; DOI : 10.4000/ephaistos.2468

Ce document a été généré automatiquement le 20 avril 2019

Tous droits réservés 


\title{
Le Chantier 1810 du Musee National des Arts et Traditions Populaires (1942-1946) : un conservatoire des techniques et de leurs gestes?
}

\author{
The 1810 Project of the MNATP (1942-1946): a Conservatory for the Technical
}

Gesture?

Bénédicte Rolland -Villemot

1 Les chantiers intellectuels lancés à la demande de l'État français constituent encore aujourd'hui une source majeure pour la recherche en ethnologie. La période de 19431945 vit l'essor des chantiers intellectuels. Pourquoi cette appellation?

2 Marcel Maget est nommé directeur du Service de la Civilisation paysanne créé en octobre 1941 par la Corporation paysanne. Ce syndicat devait contribuer au chantier de rénovation de la société française par celle de la paysannerie française. René Rémond souligne que «toutes les réformées conçues par l'Etat français, c'est sans doute celle qui exprime le plus exactement l'inspiration de la Révolution nationale comme l'ambition du maréchal Pétain de reconstruire la France sur des fondements traditionnels ${ }^{1}$ ». A partir de 1941, le Musée bénéficie de ces «chantiers intellectuels », grâce aux moyens fournis par Edmond Humeau, de la Délégation générale de l'équipement national. Des peintres et des dessinateurs, comme Jean Amblard ou René Perrot, ont la mission « d'interpréter les aspects intimes de la campagne française durant la guerre ${ }^{2}$. » Ce furent de 1941 à 1947, les chantiers EMT (enquête sur le mobilier traditionnel), EAR (enquête sur l'architecture rurale, et le chantier 1810 (son numéro administratif) sur l'artisanat.

3 Le chantier 1810, dirigé par Marcel Maget illustre la conception de la démarche empirique fondée sur l'enquête de terrain telle que le musée avait pu en organiser dès 1939. L'ethnologie folklorique pendant le régime de Vichy confirme le rôle déterminant que peuvent jouer les enquêtes dans le domaine de la recherche scientifique. 


\section{La genèse du chantier 1810} présente dans la démarche des folkloristes. L'ethnologie, science sociale qui se développe à l'époque emploie la méthode descriptive qu'est la monographie pour viser à l'exhaustivité. « On collectionnera tous les produits fabriqués ", écrit Marcel Mauss, dans son Manuel d'ethnographie, en ajoutant: «on étudiera tous les moments de la fabrication des machines. " d'une institution ou d'un fait social, la monographie désigne à la fois une méthode spécifique d'enquête et une forme d'exposition des résultats de la recherche. Elle demande une observation directe et prolongée des faits. Le premier à utiliser cette méthode fut Frédéric Le Play dans le milieu des années 1820, pour son étude des ouvriers européens. Un siècle plus tard, dans les années vingt, avec le rejet de l'ethnologie de cabinet, l'ethnologie "exotique " reprend la méthode et la développe. En 1922, Malinowski écrit sa célèbre monographie sur les Tobriandais.

En France, cet effort de méthodologie part de l'enseignement de Marcel Mauss à l'Institut d'ethnologie, entre 1928 et 1939. Chargé de l'organisation d'un service de recherche au Musée National des Arts et Traditions Populaires, entre 1943 et 1944, Marcel Maget va s'en inspirer pour définir une méthode spécifique à l'ethnographie. Il la décrit dans un texte du 18 octobre 1943 qu'il intitule « ethnographie folklorique et ethnologie ${ }^{3}$ » :

« chacun des objets étudiés ou recueillis dans les missions d'ethnographie entreprises sous la direction du musée de l'Homme, fait l'objet d'une description complète où apparaissent non seulement à côté de son nom : ses caractéristiques matérielles, dimensions, matières... mais aussi les procédés de fabrication et ses caractères économiques sociaux et idéologiques $»^{4}$.

L'artisanat et l'art populaire sont des objets d'études privilégiés de la méthode descriptive. En 1928, au Congrès de Prague, consacré aux arts populaires, Henri Focillon précise dans l'introduction de la publication consacrée à ce colloque, «la société des Nations a pensé qu'une étude méthodique des arts populaires pouvait prêter à d'intéressants échanges de vues sur les rapports qui unissent les formes de l'art ${ }^{5}$ ».

Les monographies permettent des études comparatives pour établir des rapports, des filiations ou des survivances, des traits culturels archaïques qui font appel aux notions de caractères et de transmissions populaires. Les monographies, en établissant un simple constat, doivent également fournir le substrat d'une analyse comparative et typologique. Or, c'est l'artisanat, dans le contexte de la France rurale de l'entre-deux-guerres, qui apparaît comme le meilleur objet d'étude pour faire surgir ces survivances, pour les étudier, les conserver, et les transmettre afin de développer un art populaire vivant. L'exposition internationale de Paris en 1937, était destinée entre autres buts, selon le commissaire général de l'Exposition, Edmond Labbé, à développer un thème particulier, les arts et les techniques. Les diverses appréciations de la nature du rapport entre arts et techniques vont donner lieu à différentes manifestations rattachées soit aux métiers d'art, (les objets de luxe), soit à une production essentiellement régionale : un centre des métiers, un centre rural, un centre régional pouvaient être visités lors de cette exposition.

e-Phaïstos, VI-1 2017| 2018 
9 Le centre régional fut l'un des clous de l'exposition. L'idéologie sous-jacente était alors de favoriser l'affirmation d'identités régionales à travers une même conception de l'esprit national ; il y a eu un ruralisme défendu par la gauche du Front national. Des tendances idéologiques anti-industrielles liées au régionalisme furent défendues par certains élus du Front populaire. Labbé écrit : «J'ai pour ma part confiance dans l'avenir du régionalisme, dans l'avenir de son aspect technique d'abord, c'est-à-dire de l'artisanat, j'ai entendu prouver que l'usine, faite à la mesure de la machine ne supplantera pas définitivement l'atelier, fait à la mesure de l'homme et que l'évolution des procédés de productions n'a

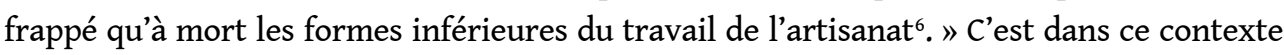
que fut organisé le Congrès international de Folklore. Lors de ce congrès, le problème de l'aménagement du musée des Arts et Traditions populaires et de la constitution des collections est évoqué. "Dans cette France, notre domaine, comment constituer nos collections ?..... Les produits et les instruments de la civilisation industrielle n'étaient pas pour nous. Dans un monde en pleine transformation, il fallait atteindre les témoins épargnés d'une civilisation artisanale arrivée à son stade ultime, mais encore florissante un siècle et demi plus tôt ${ }^{7}$ » Voilà le programme défini par Georges-Henri Rivière. La méthode monographique paraît alors la plus appropriée pour étudier la culture matérielle et plus particulièrement les techniques. Accompagnée d'enquêtes-collectes destinées à enrichir les collections du futur musée, la méthode monographique fut expérimentée, lors de deux « missions d'ethnographie folklorique » en 1938, en Bretagne et en Sologne.

\section{Description de la méthode employée pour ce chantier}

10 Le chantier 1810 est organisé en relation avec le ministère de l'Agriculture et la Corporation nationale paysanne. Il concerne " les arts et traditions populaires de la paysannerie " et utilise la méthode monographique. La monographie d'entreprise artisanale a été mise au point par Marcel Maget et enseignée dans le cours d'ethnographie française à l'école du Louvre à partir de 1941, dans le cadre du cours organique consacré à l'ethnologie de la France mis en place par Georges-Henri Rivière en 1939.

11 Entre 1942 et 1946 sous la direction de Marcel Maget, alors directeur du service de la civilisation paysanne au sein de la Corporation paysanne, 63 enquêtes ont lieu dans la France entière, mais plutôt dans la France de l'Ouest. Les enquêteurs doivent utiliser la méthode monographique qu'il a définie. Le chantier 1810 est celui qui développe le plus la méthode de l'enquête intensive. Maget va développer tout une méthodologie de la description d'objets et entreprises artisanales dans différents textes : « instruction provisoire pour la constitution d'une collection d'ethnographie française, la monographie d'objets $^{8}$ ». Ce texte conduit à la mise au point d'une fiche d' « objets folkloriques » qui doit être la méthode employée pour la description technique des objets entrant dans le musée. Dans un ordre de service du 9 mai 1941 à destination du personnel scientifique du MNATP, Maget rédige des « instructions préliminaires » à une fiche d'objet folklorique. Il développe cette notion de fiche d'objets dans une instruction du 27 octobre 1943 « dédiée au personnel et aux amis du MNATP, aux équipiers des chantiers 909, 1187, 1810 et 1425 du service des chantiers intellectuels et artistiques et aux élèves du cours d'histoire des arts et traditions populaires de l'École du Louvre ». Dans la préface, il écrit « les présentes instructions ont été élaborées au Musée de l'Homme (dès 1929), puis au MNATP ; elles ont 
été éprouvées par d'importantes missions sur le terrain (mission Dakar-Djibouti, mission de folklore musical en Basse-Bretagne) ».

Cette fiche d'objet, selon cette instruction, a deux buts : décrire les objets sur le terrain de l'enquête et servir à la rédaction de la fiche définitive d'objet quand celui-ci entre dans les collections du musée. C'est donc une monographie d'objet qui doit suivre une méthode rigoureuse : dénomination exacte de l'objet, description physique, techniques de fabrication, techniques d'utilisation, histoire du spécimen et du type ainsi que si possible des comparaisons. Les équipiers des différents chantiers doivent utiliser cette méthode pour décrire les objets ou machines qu'ils vont trouver chez les artisans. La monographie d'entreprise comprend donc des monographies d'objets ou fiches descriptives ; ce qui permet de décrire une chaîne de fabrication avec précisions.

Maget rédige également une note critique sur les outils de l'enquête. Il va également s'intéresser au rôle de l'image comme outil de connaissance et de description dans l'enquête. Ce sont des «suggestions concernant l'emploi de la photo et du cinéma dans la recherche en ethnographie paysanne ». Dans ce texte, il insiste sur le rôle important de l'image comme outil de documentation et de description indispensable à l'enquête de terrain. En 1942, dans une autre note sur les « indications psychologiques sur la méthode d'enquête à la campagne ", il met en garde sur les difficultés d'établir une relation avec son terrain de recherche et avec les personnes rencontrées. Dans son cours à l'École du Louvre en 1942-1943, il explique à ces élèves que la monographie « a pour but d'étudier sur le vif. ». Il leur donne des conseils à sur la façon de se présenter auprès des artisans qu'ils rencontrent pour obtenir des renseignements sans ni les choquer ni les froisser. Parce qu'obtenir un bon contact avec les informateurs, permet d'obtenir des renseignements fiables.

Dans le texte du 18 octobre 1943 déjà cité, Maget conclut : «Reprenant la tradition des encyclopédistes du 18ème siècle, et étendant à l'ethnographie les méthodes qui peu à peu s'élaborent et confirment leur efficacité en ethnographie, G.H Rivière a voulu que l'on entreprenne une description approfondie des techniques artisanales qu'il est encore permis d'observer sur le territoire métropolitain ». Mais plus loin, il ajoute : «nous avons insisté sur la méthode monographique, mais il ne faudrait pas en conclure de la considérer comme le seul moyen de mener une enquête intensive. Elle ne se suffit que si les points d'observations sont suffisamment rapprochés et étudiés dans un laps de temps suffisamment court pour rendre les comparaisons valables». C'est tout l'enjeu de ce chantier qui est mené dans un contexte difficile.

Cette méthode de la monographie d'entreprise artisanale doit préciser dans le cadre de chaque enquête, pour chaque entreprise ou atelier artisanal, le milieu géographique, les matières premières, les produits fabriqués, le rythme de production, le contrat social et économique, une biographie des artisans, un relevé des coutumes ainsi qu'un historique du métier. Ces éléments textuels, selon la méthode monographique, doivent être complétés par des dessins, des relevés, des croquis. Chaque enquête doit suivre la même méthode: recherche sur le terrain, préparation et élaboration d'un journal de route, chiffres, croquis, dessins mis au net éventuellement accompagnés de photographies.

Un rapport moral et un état d'avancement doivent être rédigés en trois exemplaires. Cette exigence de la méthode a influencé le choix des enquêteurs qui auront souvent une double formation. En effet, la maîtrise des techniques du dessin est un critère essentiel. Beaucoup d'enquêteurs ont donc une formation artistique. Mais cette exigence méthodologique de la monographie poursuit des buts contradictoires, en associant un 
souci d'exhaustivité à une systématique analytique. Ce genre monographique qui isole l'objet de son étude pose le problème des échelles pertinentes de l'analyse ethnologique. Cette méthode doit s'interroger sur les dimensions spécifiques du terrain et sur les problèmes de l'enquête.

\section{L'organisation du chantier}

Dans une note du 3 juin 1943, ensemble, Georges Henri Rivière et Marcel Maget expliquent les motivations qui les ont poussés à créer ces chantiers : « des circonstances sans précédent dans l'histoire du monde agissent depuis un siècle sur le développement de la civilisation occidentale. La révolution industrielle bouleverse les techniques et l'économie et sape les bases millénaires de l'ancienne société agricole et artisanale ».

\section{Les buts du chantier 1810} par un arrêté du 19 mai 1943, il n'est prévu que 14 équipiers. En réalité, par manque de crédits, le chantier ne pourra employer que 13 équipiers (12 + Maget). En 1945, un enquêteur coûtait 6500F par mois. Dans les rapports remis annuellement, on peut étudier les évolutions et les difficultés d'organisation qu'a connues ce chantier. Ainsi, à partir de mars 1943, la mise en place de la ligne de démarcation complique sérieusement les déplacements des équipiers. Dans le programme de l'année 1945-1946, des modifications sont prévues pour tenir compte des désordres induits par la Libération : «la période de transition durant laquelle la raréfaction des moyens de transport empêchant le travail d'enquêtes importantes sera utilisée à l'étude de certains artisanats de Paris ${ }^{9}$ » En ce qui concerne le type d'artisanat étudié, depuis 1942, il avait été décidé de mettre l'accent sur les métiers du bois et de la céramique, métiers traditionnels encore présents dans l'espace rural français. L'étude du corpus disponible pour le chantier 1810 aux archives nationales 
montre un l'importante diversité des métiers étudiés : vanniers de Brière, potiers de la Borne, fondeurs de Villedieu-les-Poêles, artisanats du bois, tonneliers, sabotiers.

Certains enquêteurs vont participer à plusieurs chantiers comme Soulier ou Creston. Marcel Maget lui-même effectue des enquêtes dont celle sur les dinandiers à Villedieules-Poêles. Mais celui qui va en effectuer le plus est Dan Laillier (24 enquêtes sur 63). Né à Nantes en 1919, il devient élève à l'École des beaux-arts de Nantes puis fréquente l'École du Louvre entre 1943-1945, époque où il est embauché comme enquêteur. Il participe également au chantier 909 sur le mobilier traditionnel. Il enseigne les méthodes du dessin technique aux élèves du cours d'ethnographie française de l'École du Louvre. Il étudie plus particulièrement le centre de céramique de Saint-Jean-la-Poterie qui deviendra une thèse de l'École du Louvre, dirigée par Marcel Maget. De 1954 à 1981, il est conservateur du musée de Saint-Malo ; ses dispositions en dessin lui ont permis tout au long de sa vie de mener une carrière de peintre, de dessinateur et d'illustrateur. Dan Laillier représente bien le profil de l'enquêteur du chantier qui a souvent une formation artistique.

À cette formation artistique est souvent associée une formation plus technique. Ainsi, Guy Moinet, né en 1916, élève à l'École des beaux-arts de Paris, puis professeur de l'enseignement technique et industriel, a fait 11 monographies. Il dispense à tous les équipiers des cours de dessin industriel ${ }^{10}$. Pierre Soulier est né à Paris le 23 décembre 1913 à Paris. Il est diplômé de l'Ecole nationale supérieure des arts industriels. Il effectue au sein de ce chantier l'enquête sur un éventailliste à Andeville dans l'Oise. Cette enquête fera l'objet d'un article en décembre 1944 dans le Bulletin folklorique d'Ile de France. Soulier deviendra collaborateur technique au musée puis chef du service des photographies et films du MNATP. En 1944, George-Henri Rivière décide de lancer des enquêtes ethnographiques en milieu urbain et plus particulièrement à Paris. Il demande alors à son collaborateur, Pierre Soulier d'entreprendre une étude sur les théâtres de marionnettes. Pierre Soulier commence par les théâtres parisiens, avec pour but de collecter des objets et des informations. Soulier envoie des questionnaires et ensuite effectue à Paris puis en province des enquêtes de terrain. Il a décrit sa façon de procéder dans ses « souvenirs d'enquêtes sur les théâtres de marionnettes ».

Les chargés de mission et Maget lui-même publient des articles dans l'organe de la Corporation paysanne et dans "métiers de France ", édité par le service de l'Artisanat. Les idées qui s'en dégagent sont les liens étroits entre le travail, l'art traditionnel et la société rurale. En effet, de nombreuses questions dans le cadre de l'enquête, portent sur la transmission des savoir-faire dans le cadre de la famille et de compagnonnage. Un des buts de ce chantier pour Marcel Maget est d'établir des liens entre les trois enquêtes (EAR, EMT, chantier 1810) afin de concourir à une reconnaissance et une renaissance des matériaux traditionnels dans l'architecture, à une reconnaissance des savoir-faire dans le mobilier et l'artisanat. Pour lui, un des buts de l'enquête à Villedieu-les-Poêles, est de « réunir des renseignements ayant trait aux accessoires de métal destinés aux meubles rustiques ». Dans le texte déjà cité du 18 octobre 1943, il s'inquiète de la disparition des métiers artisanaux :

« les métiers artisanaux déclinent en silence et certains disparaissent au moins localement sans laisser de trace, leur transmission est avant tout orale ; point de manuel de technologie indiquant comment on prépare des techniciens.... » 
lui, ce chantier doit avoir une mission de conservatoire des métiers d'art. Mais il ne remplira pas tous ses objectifs. De fait, il étudie surtout les métiers du bois (6 sabotiers, 7 tonneliers, 1 écorceur, 1 fendeur, soit 15 monographies), les métiers de la céramique (7 potiers). Finalement, peu d'enquêtes étudient les métiers du bâtiment ou de la petite métallurgie. C'est Boulin qui, en dehors de ce chantier, effectuera des enquêtes sur la petite métallurgie. Les régions concernées sont majoritairement situées en France du centre et de l'Ouest. La Bretagne et les Pays de la Loire représentent 34 monographies; ce sont les régions natales de Creston et Laillier. Dans le plan de travail pour l'année 1944, il est précisé que « le chantier s'est orienté vers des régions qui sans doute à cause de leur moindre importance territoriale n'ont pas encore été étudiées au point de vue qui nous occupe » c'est-à-dire du point de vue de l'artisanat. D'un point de vue pratique, on peut penser que dans le contexte de guerre, il était plus facile pour les équipiers d'enquêter dans leur région natale.

\section{L'héritage du chantier 1810}

Une volonté politique vient affirmer son soutien au développement de l'artisanat et le chantier essaie d'en être l'un des acteurs. Certaines de ses données seront utilisées par des architectes dans le cadre des premiers chantiers de la reconstruction en 1942. Louis Hautecoeur, Secrétaire général des Beaux-Arts, prononce en janvier 1943 une conférence sur le régionalisme et l'architecture, où il insiste dans le cadre des chantiers de la reconstruction sur la nécessité d'utiliser des savoir-faire artisanaux traditionnels dans le bâtiment. Ce chantier se voulait donc le lien entre tous les chantiers. Le chantier 1810 va enquêter sur des couvreurs.

Dans ce but, à certaines enquêtes sont associées des « artistes ethnographes » du chantier 1187, comme Jean Amblard. Georges Henri Rivière leur donnait comme instruction de représenter les hommes " soit à l'image d'un Courbet, livrés à des occupations spécifiques de travail ou de loisirs ; soit à l'image de Le Nain, en regardant d'un œil fixe l'observateur dans une attitude de pause étrangère à leurs activités coutumières ${ }^{11}$ ».

Sur le plan scientifique, il a permis une connaissance de certains artisanats ruraux dont témoignent encore aujourd'hui les archives du chantier conservées au musée. Ce même travail de Dan Laillier sera présenté dans l'exposition la «Bretagne, art populaire, ethnographie régionale » présentée au musée national des arts et traditions populaires du 23 juin au 23 septembre 1951. Dans l'introduction du Catalogue Georges Henri Rivière écrit : "La troisième section est en quelque sorte la description visuelle du centre céramique de Saint-Jean-La Poterie. Les photographies et les relevés dont il est l'auteur évoquent le cadre, les processus techniques et l'histoire économique de ces activités. Tableau que complètent poteries et outillages de fabrication ». Le plan de cette section de l'exposition reprend celui de la monographie d'entreprise artisanale. Dans cette exposition, comme dans le cadre du chantier, c'est la production artisanale qui est étudiée. En effet, Lallier précise que c'est le four préindustriel encore présent en 1943 qui est étudié. Le four électrique de 1946 n'est qu'évoqué. 


\section{Un conservatoire du geste technique}

31 Le but essentiel de ce chantier intellectuel, dans le cadre de la Confédération paysanne, est de participer à la reconnaissance des métiers d'artisanat « paysans ", c'est-à-dire dans un contexte rural en voie de disparition. Ce repérage sur le terrain devait servir à la transmission de savoir-faire utile pour la reconstruction nationale et la formation des artisans.

Seul le chantier 1810 applique les méthodes scientifiques et techniques de la monographie pour essayer de décrire l'action technique. Il est le seul des chantiers intellectuels à utiliser comme outil méthodologique la notion de technologie. Il a considéré ce concept comme outil d'observation, de description et d'analyse des processus techniques. Le concept de technologie tel qu'il est présent dans les travaux de Marcel Maget et surtout de Leroi-Gourhan dans les années 1940, englobe une idée sousjacente de processus technique. Si l'on veut mettre en évidence la logique interne d'une activité, il faut étudier un enchaînement d'actes, de gestes, instruments constituant un processus technique avec des étapes plus ou moins prévisibles : c'est la notion de chaîne opératoire de Leroi-Gourhan. Cette notion aboutira chez Robert Cresswell à la notion de technologie culturelle ${ }^{12}$.

Néanmoins, s'il existe bien un enchaînement obligatoire dans les grandes étapes depuis l'acquisition de la matière première jusqu'à l'usage des objets, les limites deviennent plus floues si l'on pense en terme d'actes techniques, opérations effectuées dans un temps et un espace donnés, en vue d'un usage. C'est là que les difficultés pour étudier les actes techniques commencent, cela d'autant plus que les processus techniques ne s'enchaînent pas de façon linéaire, mais s'imbriquent les uns dans les autres.

Il faut aussi étudier le savoir technique que l'artisan mobilise pour obtenir un résultat. Pour essayer de comprendre ces processus, les enquêteurs du chantier 1810, selon les recommandations du plan de la monographie d'entreprise, définissent pour chaque activité une opération technique élémentaire. On retrouve la description de cette opération élémentaire dans chaque monographie. Les enquêteurs vont donc décortiquer le processus pour arriver à l'opération élémentaire dont ils vont soigneusement décrire les phases. Cette description est le cœur des enquêtes de terrain et le noyau dur de ce qui est dénommé « technologie » dans ce plan.

Mais comment déterminer l'opération élémentaire? Le plan de la monographie propose un découpage précis : étude des lieux et des locaux et de la division du travail. Ensuite, il est proposé d'analyser le processus opératoire qui se décompose en but poursuivi, nom du processus, matière, outil et gestes employés. Puis vient la description de chaque opération élémentaire. Pour Marcel Maget, le processus opératoire se subdivise donc en plusieurs opérations élémentaires qui consistent en la position du corps, de la prise d'outil, de l'action, de l'outil ou de la main. La dernière phase de l'analyse de l'opération technique, selon le plan de la monographie, doit porter sur l'évolution, la transformation ou variantes, ainsi que l'ampleur ou le rythme de travail.

L'efficacité du geste et sa technicité sont donc difficiles à mesurer et il impossible d'en mesurer les effets. Le geste technique et le savoir-faire restent peu intelligibles. Si l'artisan expérimenté doit savoir ce qu'il fabrique, mais non pas comment il obtient ses résultats, l'activité du technicien est inspirée par des règles opérationnelles qui sont 
indépendantes de la conception du produit final. Le geste technique est plus simple à étudier quand l'artisan est polyvalent, et qu'il fait tout seul les principales opérations élémentaires dans le domaine du régime de la pratique. La parcellisation des tâches, la division du travail rendent les notions de chaînes opératoires, d'opérations élémentaires, beaucoup moins opérationnelles. Cet ensemble de monographies est bien un alphabet du geste technique. Mais cet alphabet ne s'exprime pas par le langage, ni parlé, ni écrit. C'est par la communication visuelle, dessins, photographies..., que se comprend le geste. Son apprentissage et sa transmission au sein des ateliers se fait aussi par une communication visuelle. C'est par immersion et en regardant que l'apprenti comprend ce qu'il doit faire.

Un des buts principal de cet ensemble de 63 monographies est d'étudier, de la technique à l'objet, le geste à l'œuvre, et de l'objet à la technique, quels sont les gestes à l'œuvre. L'étude du geste effectué, de l'outil employé et de la position du corps occupent une place centrale dans la description de la monographie. C'est évidemment le geste de la main qui est détaillé pour essayer de comprendre si c'est l'outil qui est conditionné à l'origine par le geste à faire ou si l'outil conditionne le geste à faire. L'enquêteur essaie donc de déterminer quel est l'outil principal pour chaque geste. Et ce sont toujours les opérations faites à la main qui sont décrites et dessinées. Comme la méthode employée repose essentiellement sur la synchronie, la description du geste repose sur une analyse visuelle de la synchronie sans tenir compte ni de l'anatomie et de la physiologie. Se pose alors la question du degré d'exhaustivité de la description puisque la méthode ne définit pas les éléments pertinents à analyser pour l'étude gestuelle.

Il semble plus prudent, en effet, dans un premier stade de description d'un geste technique, de ne pas opérer un choix parmi les membres du corps à décrire, c'est-à-dire de ne pas se limiter aux parties du corps qui paraissent les plus actives, les plus immédiatement fonctionnelles. Or, nous l'avons vu, les enquêteurs du chantier 1810 privilégient la main comme le continuum du cerveau et dont l'outil est le prolongement. Mais si l'on considère, avec André -Georges Haudricourt, que « l'évolution de l'outil ne s'explique que par une constante adaptation aux différentes techniques et aux différents besoins ${ }^{13}$ ", on peut s'interroger sur l'intérêt des études centrées sur le processus opératoire générique, sur la diachronie technique. Ce serait, en effet, ces mouvements, les variantes, qui seraient les plus aptes à expliquer la filiation généalogique des opérations techniques et de leurs produits. Il faut alors concevoir l'outil comme support d'une innovation technique. Diachronie-synchronie, ce mouvement dialectique est au cœur de ces techniques de description de l'outil et de son complexe technique.

Constituer des archives de la technique est le but explicitement revendiqué par Marcel Maget dans les notes et directives qu'il donne aux enquêteurs sur le terrain. Ce but est d'autant plus assigné au chantier 1810 qu'il doit contribuer à l'enrichissement des collections du musée national des arts et traditions populaires en construction. Ce but ne va être rempli que de façon partielle. En effet, comme trop peu d'ateliers et de lieux de travail vont être étudiés, il va être difficile de constituer des archives de la technique complètes et représentatives. De plus, ce chantier 1810 connaît une difficulté méthodologique et épistémologique: il est tiraillé entre la monographie intensive décrire à fond un atelier - et la monographie extensive - décrire les activités humaines sur un territoire, la Basse-Bretagne. Il est partagé entre la diachronie et la synchronie. Il voudrait rendre compte d'un fait social total. Mais n'est-ce pas une utopie?

Quant au geste technique, il n'a pas pour seule finalité de produire un objet. Toute technicité incorpore la culture technique d'un individu ou d'un groupe, qui sont 
difficilement appréhendables sur le terrain et qui ne sont pas objets de musée. Car apprendre une technique, c'est aussi un travail sur soi. Avoir le geste rapide et sûr demande des mois d'apprentissage pendant lesquels le sujet se modifie progressivement par son action sur la matière. Conserver et transmettre un savoir-faire en l'inscrivant dans une technologie culturelle, n'est-ce pas aussi une utopie?

\section{BIBLIOGRAPHIE}

BAFFET Hélène (dir.), Observer l'action technique : des chaînes opératoires pour quoi faire?, Paris, CNRS, 1991

BRIL Blandine, «Description du geste technique : Quelles méthodes? ", Techniques \& Culture [En ligne], 54-55 | 2010, mis en ligne le 30 juin 2013

GARÇON Anne-Françoise, L'imaginaire et la pensée technique, une approche historique XVI ${ }^{e}-X I X^{e}$, Paris, Garnier, 2012

GARÇON Anne-Françoise, « Des modes d'existence du geste technique », e-Phaïstos, IV-2, 2015, p.84-92, mis en ligne le 29 novembre 2016. URL : http://journals.openedition.org/ephaistos/797

INGOLD Tim, « L'Outil, l'esprit et la machine : une excursion dans la philosophie de la "technologie"», Techniques \& Culture [En ligne], 54-55 | 2010, mis en ligne le 30 janvier 2013

LEROI-GOURHAN André, L'homme et la matière, Paris, Albin Michel, 1943

LEROI-GOURHAN André, Milieu et techniques, Paris, Albin Michel, 1943 et 1971

MAUSS Marcel, «Les techniques du corps », [article originalement publié dans le Journal de Psychologie, XXXII, 3-4, 15 mars - 15 avril, 1936.] «Les techniques du corps » Un document produit en version numérique par Jean-Marie Tremblay, professeur de sociologie au CÉGEP de Chicoutimi dans le cadre de la collection: « Les classiques des sciences sociales » «Sitewebhttp:// www.uqac.uquebec.ca/zone30/Classiques_des_sciences_sociales/ind ex.html

RIVIÈRE Georges Henri, «Collections d'objets et documents d'ethnographie française », Bulletin des musées de France, 1946, p. 26-34

\section{NOTES}

1. RÉMOND René, in BOUSSARD Isabel, Vichy et la Corporation paysanne, Paris, Presses de la Fondation nationale des sciences politiques, 1980, p.11.

2. RIVIÈRE Georges -Henri, « Musée national des Arts et traditions populaires ", Musées nationaux, Nouvelles acquisition, 2 septembre 1939-2 septembre 1945, Paris, 1945, p. 99-103.

3. Archives Nationales, archives Maget.

4. Ibidem.

5. Actes du Colloque d'arts populaires de Prague, Paris, 1929, p. 8.

6. Institut Français d'architecture, Pour le cinquantenaire de l'exposition internationale de 1937, Paris 1987, p.36. 


\section{Ibidem.}

8. Archives Nationales, Fonds Maget, 48-21

9. Il s'agit de l'enquête sur l'entreprise Hermès effectuée par Moinet.

10. Archives Nationales, Monographie 20130277/115.

11. RIVIÈRE Georges -Henri, "Nature et fonctionnements des chantiers intellectuels ", in Vingt dessins de Jean Amblard, Musée d'art et d'histoire de la ville de Saint-Denis, Saint-Denis, 1982, p. 8-9.

12. FABRE Daniel, «Introduction », Vers une ethnologie du présent, Paris, Éditions de la Maison des sciences de l'homme, 1996, Disponible sur Internet: <http://books.openedition.org/ editionsmsh/3873>. DOI : 10.4000/books.editionsmsh.3873.

13. HAUDRICOURT André-George, La technologie, science humaine. Recherches d'histoire et d'ethnologie des techniques, Paris, éditions de la Maison des sciences de l'homme, 1987, p. 37-46.

\section{RÉSUMÉS}

Comment décrire les gestes techniques pour la préservation du Patrimoine, ou pour leur valorisation et leur transmission? Comment même expliquer les gestes techniques? Et avec quels moyens intellectuels? L'étude des monographies des entreprises artisanales françaises réalisées dans le cadre du «projet intellectuel 1810 » mené par l'équipe du Musée National des Arts et Traditions Populaires (MNATP) entre 1940 et 1946 nous donne une réponse historique à la question. La méthode de la monographie suivie des collectes-enquêtes destinées à enrichir le nouveau musée semble alors être la plus adaptée à l'étude de la culture matérielle et surtout des techniques. Le but de cet article est d'analyser les contributions et les limites de cette méthode ethnographique à visée muséologique.

How to describe technical gestures for the preservation of the Heritage, or for their valuation and their transmission? How even explain the technical gestures? And with which intellectual means? The study of the monographs of french craft enterprises made within the context of the "intellectual project 1810" conducted by the team of the National Museum of Popular Arts and Traditions (MNATP) between 1940 and 1946 give us an historical answer to the question. The monograph method followed-up of collecting-inquiries intended to enrich the new museum seemed then to be the most suited to study the material culture and especially the techniques. The purpose of this article is to analyze the contributions and limitations of this ethnographic method aimed for museological purposes.

\section{INDEX}

Mots-clés : histoire des techniques, France, années 1940, geste technique, enquête ethnographique, monographie d'entreprise, artisanat, chaîne opératoire

Index chronologique : Époque contemporaine

Index géographique : France

Keywords : history of technology, France, 1940s, technical gesture, ethnographic inquiry, craftsmanship 


\section{AUTEUR}

\section{BÉNÉDICTE ROLLAND -VILLEMOT}

Conservateur en chef du patrimoine, Bénédicte Rolland -Villemot est en charge des musées de société et des musées techniques au service des Musées de France au ministère de la Culture. Elle prépare une thèse au Centre d'Histoire des Techniques de l'Université Paris 1 PanthéonSorbonne (IHMC UMR CNRS 8066), sur l'Histoire des techniques, matières et matériaux dans la pratique des musées de France des années 1960 aux années 2000. Elle est enseignante à L'Ecole du Louvre et à l'institut national du patrimoine. Ses travaux portent sur l'histoire matérielle des collections de musées, sur les rapports en l'histoire des techniques et les pratiques muséales et sur les collections muséales comme archives matérielle pour une histoire de techniques. 